Gustavo Jardim de Morais

Análise da Teoria dos Valores Extremos e da Simulação de Monte Carlo para o cálculo do Value-at-Risk em carteiras de investimentos de ativos de renda variável

Dissertação apresentada como requisito parcial para obtenção do grau de Mestre pelo Programa de PósGraduação em Engenharia de Produção do Departamento de Engenharia Industrial da PUC-Rio.

Orientador: Prof. Carlos Patrício Samanez 
Gustavo Jardim de Morais

\section{Análise da Teoria dos Valores Extremos e da Simulação de Monte Carlo para o cálculo do Value-at-Risk em carteiras de investimentos de ativos de renda variável.}

Dissertação apresentada como requisito parcial para obtenção do grau de Mestre pelo Programa de PósGraduação em Engenharia de Produção do Departamento de Engenharia Industrial da PUC-Rio. Aprovada pela Comissão Examinadora abaixo assinada.

Prof. Carlos Patrício Samanez

Orientador

Departamento de Engenharia Industrial - PUC-Rio

ProfessorJosé Paulo Texeira

Departamento de Engenharia Industrial - PUC-Rio

Professor Fábio Rodrigo Siqueira Batista

Departamento de Engenharia Industrial - PUC-Rio

Professor José Eugênio Leal

Coordenador Setorial do Centro

Técnico Científico - PUC - Rio.

Rio de Janeiro, 14 de Abril de 2011 
Todos os direitos reservados. É proibida a reprodução total ou parcial do trabalho sem autorização da Universidade, do autor e do orientador.

\section{Gustavo Jardim de Morais}

Graduado em Engenharia de Produção Elétrica e Engenharia Elétrica com Ênfase em Sistemas de Apoio à Decisão na Pontifícia Universidade Católica do Rio de Janeiro em 2007. Após estágio no mercado financeiro e trabalhar como engenheiro projetista, retornou mercado financeiro para um Banco de Investimentos. Fez cursos na área no Brasil e em Londres.

Ficha Catalográfica

Morais, Gustavo Jardim de

Análise da teoria dos valores extremos e da simulação de Monte Carlo para o cálculo do value-at-risk em carteiras de investimentos de ativos de renda variável / Gustavo Jardim de Morais ; orientador: Carlos Patrício Samanez. 2010.

225 f. : il. (color.) ; $30 \mathrm{~cm}$

Dissertação (mestrado)-Pontifícia Universidade Católica do Rio de Janeiro, Departamento de Engenharia Industrial, 2010.

Inclui bibliografia

1. Engenharia Industrial - Teses. 2. Valor em risco. 3. Teoria dos valores extremos. 4. Simulação de Monte Carlo. 5. Medida Ômega I. Samanez, Carlos Patrício. II. Pontifícia Universidade Católica do Rio de Janeiro. Departamento de Engenharia Industrial. III. Título. 
Para minha família, obrigado por todo o incentivo!

Obrigado também aos meus professores da Puc-Rio, aos colegas de turma e funcionários, e à Capes, pelo apoio financeiro e investimento em pesquisa!

"If I have seen further, it is by standing on the shoulders of giants" Sir Isaac Newton 


\section{Resumo}

Morais, Gustavo Jardim; Samanez, Carlos Patrício. Análise da Teoria dos Valores Extremos e da Simulação de Monte Carlo para o cálculo do Value-at-Risk em carteiras de investimentos de ativos de renda variável. Rio de Janeiro, RJ, 2010. 225p. Dissertação de Mestrado - Departamento de Engenharia Industrial, Pontifícia Universidade Católica do Rio de Janeiro.

Após as recentes crises financeiras que se abateram sobre os mercados financeiros de todo o mundo, com mais propriedade a de 2008/2009, mas ainda a crise no Leste Europeu em Julho/2007, a moratória Russa em Outubro/1998, e, no âmbito nacional, a mudança no regime cambial brasileiro, em Janeiro/1999, as instituições financeiras incorreram em grandes perdas em cada um desses eventos e uma das principais questões levantadas acerca dos modelos financeiros diziam respeito ao gerenciamento de risco. Os diversos métodos de cálculo do Value-atrisk, bem como as simulações e cenários traçados por analistas não puderam prever sua magnitude nem tampouco evitar que a crise se agravasse. Em função disso, proponho-me à questão de estudar os sistemas de gerenciamento de risco financeiro, na medida em que este pode e deve ser aprimorado, sob pena de catástrofes financeiras ainda maiores. Embora seu conteúdo se mostre tão vasto na literatura, as metodologias para cálculo de valor em risco não são exatas e livres de falhas. Nesse contexto, coloca-se necessário o desenvolvimento e aprimoramento de ferramentas de gestão de risco que sejam capazes de auxiliar na melhor alocação dos recursos disponíveis, avaliando o nível de risco à que um investimento está exposto e sua compatibilidade com seu retorno esperado.

\section{Palavras - chave}

Valor em risco; Teoria dos Valores Extremos; Simulação de Monte Carlo; Medida Ômega. 


\section{Abstract}

Morais, Gustavo Jardim; Samanez, Carlos Patrício (Advisor). Analysis of Extreme Values Theory and Monte Carlo Simulation for the calculation of value-at-risk in stock portfolios. Rio de Janeiro, RJ, 2010. 225p. MSc. Dissertation - Departamento de Engenharia Industrial, Pontifícia Universidade Católica do Rio de Janeiro.

After recent financial crisis that have hit financial markets all around the world, with more property on 2008/2009 periods, the Eastern Europe crisis in 2007, the Russian moratorium on October/1998, and with Brazilian national exchange rate regime change on January/1999, financial institutions have incurred in large losses on each of these events and one of the main question raised about the financial models related to risk management. The Value-at-Risk management and its many forms to calculate it, as well as the simulations and scenarios predicted by analysts could not predict its magnitude or prevent crisis worsened. As a result, I intent to study the question of financial systems management, in order to improve the existing methods, under the threat that even bigger financial disasters are shall overcome. Although it's content is vast on scientific literature, the Value-at-Risk calculate is not exact and free of flaws. In this context, there is need for the development and improvement of risk management tools that are able to assist in a better asset equities allocation of resources, equalizing the risk level of an investment and it's return.

\section{Keywords}

Value at Risk; Extreme Value Theory; Omega Theory, Asset allocation management. 


\section{Sumário}

1. Introdução 32

1.1 O surgimento dos modelos de gestão de risco financeiro 33

1.2 Revisão histórica 35

$\begin{array}{lll}1.3 & \text { Motivação } & 37\end{array}$

1.4 Estrutura do trabalho 39

2. O value-at-risk 42

2.1 Definição 42

2.2 Definição do var 44

$\begin{array}{lll}2.3 & \text { Critérios para o backtesting } & 46\end{array}$

2.4 Validação do modelo 46

2.5 O VaR simulado 48

2.6 As limitações do VaR e o CVaR 49

3. A construção das carteiras de investimento 51

3.1 A teoria das carteiras $\quad 51$

3.2 Retorno e variância de uma carteira 52

3.3 A seleção da carteira ótima 52

3.4 O modelo de índice único 53

3.5 A seleção da carteira ótima no contexto do modelo

de índice único 56

3.6 Uso da medida ômega para montar uma carteira otimizada de ativos

4. Metodologias para mensuração do valor em risco 64

4.1 A teoria dos valores extremos (t.v.e.) 64

4.2 O teorema de fischer - tippet 67

4.3 Estimação dos parâmetros da distribuição de valores extremos 68

$\begin{array}{lll}4.4 & \text { A simulação de monte carlo } & 75\end{array}$ 
5. Construção das carteiras de investimentos 81

5.1 Carteira A 84

5.2 Carteira B 88

$\begin{array}{lll}5.3 & \text { Carteira C } & 90\end{array}$

5.4 Carteira D 93

5.5 Carteira E 96

5.6 Metodologia para o cálculo da carteira teórica do lbovespa 99

6. Análise do Value - at - Risk 101

6.1 Carteira A 102

6.2 Carteira B 110

6.3 Carteira C 115

6.4 Carteira D 121

6.5 Carteira E 126

6.6 Análise do backtesting 131

7. Conclusão 133

8. Bibliografia 146

9. Apêndice A: simulações de VaR por s.m.c e t.v.e. para carteiras constituídas por M.I.U. e M.O.

10. Apêndice B: Hill - plot das carteiras de investimento calculadas por M.I.U. e M.O. 


\section{Lista de figuras}

Figura 1 - Fronteira eficiente de Markowitz

Figura 2 - Análise da performance de 2 carteiras

Figura 3 - Distribuição de retornos com $L=1,4$

Figura 4 - Distribuição de probabilidades

Figura 5 - Distribuição de probabilidades cumulativa

Figura 6 - Distribuição de frequência

Figura 7 - VaR - Carteira A.M.I.U.T.V.E. $-p=0,50 \%$

151

Figura 8 - VaR - Carteira A.M.I.U.S.M.C. $-p=0,50 \%$

151

Figura $9-$ VaR - Carteira A.M.I.U.T.V.E. $-p=1 \%$

Figura $10-$ VaR - Carteira A.M.I.U.S.M.C. $-p=1 \%$

Figura 11 - VaR - Carteira A.M.I.U.T.V.E. $-p=2,5 \%$

152

Figura $12-$ VaR - Carteira A.M.I.U.S.M.C. $-p=2,5 \%$

152

Figura $13-$ VaR - Carteira A.M.I.U.T.V.E. $-p=5 \%$

Figura $14-$ VaR - Carteira A.M.I.U.S.M.C. $-p=5 \%$

Figura $15-$ VaR - Carteira A.M.O.L. $=0 \%$.T.V.E. $-p=0,50 \%$

153

Figura $16-$ VaR - Carteira A.M.O.L. $=0 \%$.S.M.C. $-p=0,50 \%$

Figura 17 - VaR - Carteira A.M.O.L. $=0 \%$.T.V.E. $-p=1 \%$

Figura $18-$ VaR - Carteira A.M.O.L. $=0 \%$.S.M.C. $-p=1 \%$

Figura $19-$ VaR - Carteira A.M.O.L. $=0 \%$.T.V.E. $-p=2,5 \%$ 
Figura $20-$ VaR - Carteira A.M.O.L. $=0 \%$.S.M.C. $-p=2,5 \%$

Figura $21-$ VaR - Carteira A.M.O.L. $=0 \%$.T.V.E. $-p=5 \%$

154

Figura $22-$ VaR - Carteira A.M.O.L. $=0 \%$.S.M.C. $-p=5 \%$

154

Figura $23-$ VaR - Carteira A.M.O.L. $=2,5 \%$.T.V.E. $-p=0,5 \%$

155

Figura $24-$ VaR - Carteira A.M.O.L. $=2,5 \%$.S.M.C. $-p=0,5 \%$ 155

Figura $25-$ VaR - Carteira A.M.O.L. $=2,5 \%$.T.V.E. $-p=1 \%$

155

Figura $26-$ VaR - Carteira A.M.O.L. $=2,5 \%$.S.M.C. $-p=1 \%$

Figura $27-$ VaR - Carteira A.M.O.L. $=2,5 \%$.T.V.E. $-p=2,5 \%$

156

Figura $28-$ VaR - Carteira A.M.O.L. $=2,5 \%$.S.M.C. $-p=2,5 \%$

156

Figura $29-$ VaR - Carteira A.M.O.L. $=2,5 \%$.T.V.E. $-p=5 \%$

156

Figura $30-$ VaR - Carteira A.M.O.L. $=2,5 \%$.S.M.C. $-p=5 \%$ 156

Figura $31-$ VaR - Carteira A.M.O.L. $=5 \%$.T.V.E. $-p=0,5 \%$

157

Figura $32-$ VaR - Carteira A.M.O.L. $=5 \%$.S.M.C. $-p=0,5 \%$

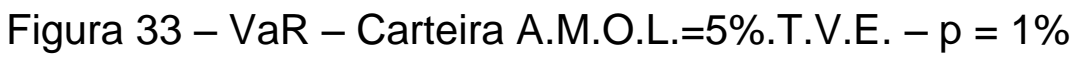

Figura $34-$ VaR - Carteira A.M.O.L. $=5 \%$.S.M.C. $-p=1 \%$

Figura $35-$ VaR - Carteira A.M.O.L. $=5 \%$.T.V.E. $-p=2,5 \%$ 158

Figura $36-$ VaR - Carteira A.M.O.L. $=5 \%$.S.M.C. $-p=2,5 \%$ 158

Figura $37-$ VaR - Carteira A.M.O.L. $=5 \%$.T.V.E. $-p=5 \%$ 158

Figura $38-$ VaR - Carteira A.M.O.L. $=5 \%$.S.M.C. $-p=5 \%$ 158

Figura $39-$ VaR - Carteira B.M.I.U.T.V.E. $-p=0,50 \%$ 159 
Figura 40 - VaR - Carteira B.M.I.U.S.M.C. $-p=0,50 \%$

Figura 41 - VaR - Carteira A|B.M.I.U.T.V.E. $-p=1 \%$

159

Figura $42-$ VaR - Carteira B.M.I.U.S.M.C. $-p=1 \%$

159

Figura 43 - VaR - Carteira B.M.I.U.T.V.E. $-p=2,5 \%$

160

Figura $44-$ VaR - Carteira B.M.I.U.S.M.C. $-p=2,5 \%$

160

Figura $45-$ VaR - Carteira B.M.I.U.T.V.E. $-p=5 \%$

160

Figura $46-$ VaR - Carteira B.M.I.U.S.M.C. $-p=5 \%$

160

Figura $47-$ VaR - Carteira B.M.O.L. $=0 \%$.T.V.E. $-p=0,50 \%$

161

Figura $48-$ VaR - Carteira B.M.O.L. $=0 \%$.S.M.C. $-p=0,50 \%$

161

Figura $49-$ VaR - Carteira B.M.O.L. $=0 \%$.T.V.E. $-p=1 \%$

161

Figura $50-$ VaR - Carteira B.M.O.L. $=0 \%$.S.M.C. $-p=1 \%$

Figura $51-$ VaR - Carteira B.M.O.L. $=0 \%$.T.V.E. $-p=2,5 \%$

162

Figura $52-$ VaR - Carteira B.M.O.L. $=0 \%$.S.M.C. $-p=2,5 \%$

162

Figura $53-$ VaR - Carteira B.M.O.L. $=0 \%$.T.V.E. $-p=5 \%$

162

Figura $54-$ VaR - Carteira B.M.O.L. $=0 \%$.S.M.C. $-p=5 \%$

162

Figura $55-$ VaR - Carteira B.M.O.L. $=2,5 \%$.T.V.E. $-p=0,5 \%$

163

Figura $56-$ VaR - Carteira B.M.O.L. $=2,5 \%$.S.M.C. $-p=0,5 \%$

163

Figura $57-$ VaR - Carteira B.M.O.L. $=2,5 \%$.T.V.E. $-p=1 \%$

Figura $58-$ VaR - Carteira B.M.O.L. $=2,5 \%$.S.M.C. $-p=1 \%$

Figura 59 - VaR - Carteira B.M.O.L. $=2,5 \%$.T.V.E. $-p=2,5 \%$ 
Figura 60 - VaR - Carteira B.M.O.L. $=2,5 \%$.S.M.C. $-p=2,5 \%$

Figura $61-$ VaR - Carteira B.M.O.L. $=2,5 \%$.T.V.E. $-p=5 \%$

164

Figura $62-$ VaR - Carteira B.M.O.L. $=2,5 \%$.S.M.C. $-p=5 \%$ 164

Figura $63-$ VaR - Carteira B.M.O.L. $=5 \%$.T.V.E. $-p=0,5 \%$ 165

Figura $64-$ VaR - Carteira B.M.O.L. $=5 \%$.S.M.C. $-p=0,5 \%$ 165

Figura $65-$ VaR - Carteira B.M.O.L. $=5 \%$.T.V.E. $-p=1 \%$ 165

Figura $66-$ VaR - Carteira B.M.O.L. $=5 \%$.S.M.C. $-p=1 \%$ 165

Figura $67-$ VaR - Carteira B.M.O.L. $=5 \%$.T.V.E. $-p=2,5 \%$ 166

Figura $68-$ VaR - Carteira B.M.O.L. $=5 \%$.S.M.C. $-p=2,5 \%$ 166

Figura $69-$ VaR - Carteira B.M.O.L. $=5 \%$.T.V.E. $-p=5 \%$ 166

Figura $70-$ VaR - Carteira B.M.O.L. $=5 \%$.S.M.C. $-p=5 \%$ 166

Figura 71 - VaR - Carteira C.M.I.U.T.V.E. $-p=0,50 \%$ 167

Figura $72-$ VaR - Carteira C.M.I.U.S.M.C. $-p=0,50 \%$ 167

Figura $73-$ VaR - Carteira C.M.I.U.T.V.E. $-p=1 \%$ 167

Figura $74-$ VaR - Carteira C.M.I.U.S.M.C. $-p=1 \%$ 167

Figura 75 - VaR - Carteira C.M.I.U.T.V.E. $-p=2,5 \%$ 168

Figura $76-$ VaR - Carteira C.M.I.U.S.M.C. $-p=2,5 \%$ 168

Figura $77-$ VaR - Carteira C.M.I.U.T.V.E. $-p=5 \%$ 168

Figura $78-$ VaR - Carteira C.M.I.U.S.M.C. $-p=5 \%$ 168

Figura $79-$ VaR - Carteira C.M.O.L. $=0 \%$.T.V.E. $-p=0,50 \%$ 169 
Figura $80-$ VaR - Carteira C.M.O.L. $=0 \% . S . M . C . ~-p=0,50 \%$

Figura $81-$ VaR - Carteira C.M.O.L. $=0 \%$.T.V.E. $-p=1 \%$

169

Figura $82-$ VaR - Carteira C.M.O.L. $=0 \%$.S.M.C. $-p=1 \%$

169

Figura $83-$ VaR - Carteira C.M.O.L. $=0 \%$.T.V.E. $-p=2,5 \%$

170

Figura $84-$ VaR - Carteira C.M.O.L. $=0 \%$.S.M.C. $-p=2,5 \%$

170

Figura $85-$ VaR - Carteira C.M.O.L. $=0 \%$.T.V.E. $-p=5 \%$

170

Figura $86-$ VaR - Carteira C.M.O.L. $=0 \%$.S.M.C. $-p=5 \%$

170

Figura $87-$ VaR - Carteira C.M.O.L. $=2,5 \%$.T.V.E. $-p=0,5 \%$

Figura $88-$ VaR - Carteira C.M.O.L. $=2,5 \%$.S.M.C. $-p=0,5 \%$

171

Figura $89-$ VaR - Carteira C.M.O.L. $=2,5 \%$.T.V.E. $-p=1 \%$

171

Figura $90-$ VaR - Carteira C.M.O.L. $=2,5 \%$.S.M.C. $-p=1 \%$

Figura $91-$ VaR - Carteira C.M.O.L. $=2,5 \%$.T.V.E. $-p=2,5 \%$

172

Figura $92-$ VaR - Carteira C.M.O.L. $=2,5 \%$.S.M.C. $-p=2,5 \%$

172

Figura $93-$ VaR - Carteira C.M.O.L. $=2,5 \%$.T.V.E. $-p=5 \%$

172

Figura $94-$ VaR - Carteira C.M.O.L. $=2,5 \%$.S.M.C. $-p=5 \%$

172

Figura $95-$ VaR - Carteira C.M.O.L. $=5 \% . T . V . E . ~-p=0,5 \%$

173

Figura $96-$ VaR - Carteira C.M.O.L. $=5 \%$.S.M.C. $-p=0,5 \%$

Figura $97-$ VaR - Carteira C.M.O.L. $=5 \% . T . V . E . ~-p=1 \%$

Figura $98-$ VaR - Carteira C.M.O.L. $=5 \%$.S.M.C. $-p=1 \%$

173

Figura $99-$ VaR - Carteira C.M.O.L. $=5 \% . T . V . E . ~-p=2,5 \%$ 
Figura $100-$ VaR - Carteira C.M.O.L. $=5 \%$.S.M.C. $-p=2,5 \%$

Figura 101- VaR - Carteira C.M.O.L. $=5 \%$.T.V.E. $-p=5 \%$

174

Figura $102-$ VaR - Carteira C.M.O.L. $=5 \%$.S.M.C. $-p=5 \%$

174

Figura $103-$ VaR - Carteira D.M.I.U.T.V.E. $-p=0,50 \%$

175

Figura $104-$ VaR - Carteira D.M.I.U.S.M.C. $-p=0,50 \%$

175

Figura $105-$ VaR - Carteira D.M.I.U.T.V.E. $-p=1 \%$

175

Figura $106-$ VaR - Carteira D.M.I.U.S.M.C. $-p=1 \%$

175

Figura 107 - VaR - Carteira D.M.I.U.T.V.E. $-p=2,5 \%$

176

Figura $108-$ VaR - Carteira D.M.I.U.S.M.C. $-p=2,5 \%$

176

Figura $109-$ VaR - Carteira D.M.I.U.T.V.E. $-p=5 \%$

176

Figura $110-$ VaR - Carteira D.M.I.U.S.M.C. $-p=5 \%$

176

Figura $111-$ VaR - Carteira D.M.O.L. $=0 \%$.T.V.E. $-p=0,50 \%$

Figura $112-$ VaR - Carteira D.M.O.L. $=0 \%$.S.M.C. $-p=0,50 \%$

Figura $113-$ VaR - Carteira D.M.O.L. $=0 \%$.T.V.E. $-p=1 \%$

Figura $114-$ VaR - Carteira D.M.O.L. $=0 \%$.S.M.C. $-p=1 \%$

Figura $115-$ VaR - Carteira D.M.O.L. $=0 \%$.T.V.E. $-p=2,5 \%$

178

Figura $116-$ VaR - Carteira D.M.O.L. $=0 \%$.S.M.C. $-p=2,5 \%$

178

Figura $117-$ VaR - Carteira D.M.O.L. $=0 \%$.T.V.E. $-p=5 \%$ 178

Figura $118-$ VaR - Carteira D.M.O.L. $=0 \%$.S.M.C. $-p=5 \%$ 178

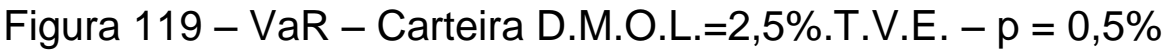


Figura 120 - VaR - Carteira D.M.O.L. $=2,5 \%$.S.M.C. $-p=0,5 \%$

Figura 121 - VaR - Carteira D.M.O.L. $=2,5 \% . T . V . E . ~-p=1 \%$

179

Figura 122 - VaR - Carteira D.M.O.L.=2,5\%.S.M.C. $-p=1 \%$

179

Figura 123 - VaR - Carteira D.M.O.L.=2,5\%.T.V.E. $-p=2,5 \%$

180

Figura 124 - VaR - Carteira D.M.O.L.=2,5\%.S.M.C. $-p=2,5 \%$

180

Figura $125-$ VaR - Carteira D.M.O.L.=2,5\%.T.V.E. $-p=5 \%$

180

Figura 126 - VaR - Carteira D.M.O.L.=2,5\%.S.M.C. $-p=5 \%$

180

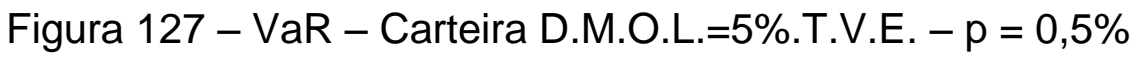

181

Figura $128-$ VaR - Carteira D.M.O.L. $=5 \% . S . M . C . ~-p=0,5 \%$

181

Figura 129 - VaR - Carteira D.M.O.L.=5\%.T.V.E. $-p=1 \%$

181

Figura 130 - VaR - Carteira D.M.O.L. $=5 \% . S . M . C . ~-p=1 \%$

Figura $131-$ VaR - Carteira D.M.O.L.=5\%.T.V.E. $-p=2,5 \%$

182

Figura $132-$ VaR - Carteira D.M.O.L. $=5 \% . S . M . C . ~-p=2,5 \%$

182

Figura 133- VaR - Carteira D.M.O.L. $=5 \%$.T.V.E. $-p=5 \%$

182

Figura $134-$ VaR - Carteira D.M.O.L. $=5 \%$.S.M.C. $-p=5 \%$

182

Figura 135 - VaR - Carteira E.M.I.U.T.V.E. $-p=0,50 \%$

183

Figura 136 - VaR - Carteira E.M.I.U.S.M.C. $-p=0,50 \%$ 183

Figura 137 - VaR - Carteira E.M.I.U.T.V.E. $-p=1 \%$ 183

Figura 138 - VaR - Carteira E.M.I.U.S.M.C. $-p=1 \%$ 183

Figura 139 - VaR - Carteira E.M.I.U.T.V.E. $-p=2,5 \%$ 
Figura $140-$ VaR - Carteira E.M.I.U.S.M.C. $-p=2,5 \%$

Figura $141-$ VaR - Carteira E.M.I.U.T.V.E. $-p=5 \%$

184

Figura $142-$ VaR - Carteira E.M.I.U.S.M.C. $-p=5 \%$

184

Figura $143-$ VaR - Carteira E.M.O.L. $=0 \%$.T.V.E. $-p=0,50 \%$

185

Figura $144-$ VaR - Carteira E.M.O.L. $=0 \% . S . M . C . ~-p=0,50 \%$ 185

Figura $145-$ VaR - Carteira E.M.O.L. $=0 \%$.T.V.E. $-p=1 \%$ 185

Figura $146-$ VaR - Carteira E.M.O.L. $=0 \%$.S.M.C. $-p=1 \%$ 185

Figura $147-$ VaR - Carteira E.M.O.L. $=0 \%$.T.V.E. $-p=2,5 \%$ 186

Figura $148-$ VaR - Carteira E.M.O.L. $=0 \%$.S.M.C. $-p=2,5 \%$ 186

Figura $149-$ VaR - Carteira E.M.O.L. $=0 \%$.T.V.E. $-p=5 \%$ 186

Figura $150-$ VaR - Carteira E.M.O.L. $=0 \% . S . M . C . ~-p=5 \%$ 186

Figura $151-$ VaR - Carteira E.M.O.L. $=2,5 \%$.T.V.E. $-p=0,5 \%$ 187

Figura $152-$ VaR - Carteira E.M.O.L. $=2,5 \%$.S.M.C. $-p=0,5 \%$ 187

Figura $153-$ VaR - Carteira E.M.O.L. $=2,5 \%$.T.V.E. $-p=1 \%$ 187

Figura $154-$ VaR - Carteira E.M.O.L. $=2,5 \%$.S.M.C. $-p=1 \%$

Figura $155-$ VaR - Carteira E.M.O.L. $=2,5 \%$.T.V.E. $-p=2,5 \%$ 188

Figura 156 - VaR - Carteira E.M.O.L. $=2,5 \%$.S.M.C. $-p=2,5 \%$ 188

Figura $157-$ VaR - Carteira E.M.O.L. $=2,5 \%$.T.V.E. $-p=5 \%$ 188

Figura $158-$ VaR - Carteira E.M.O.L. $=2,5 \%$.S.M.C. $-p=5 \%$ 188

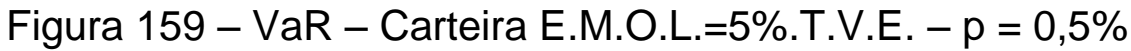


Figura $160-$ VaR - Carteira E.M.O.L. $=5 \%$.S.M.C. $-p=0,5 \%$

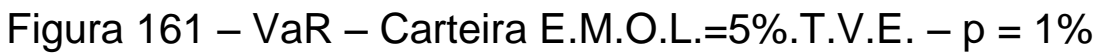

189

Figura $162-$ VaR - Carteira E.M.O.L. $=5 \%$.S.M.C. $-p=1 \%$

189

Figura $163-$ VaR - Carteira E.M.O.L. $=5 \%$.T.V.E. $-p=2,5 \%$

190

Figura $164-$ VaR - Carteira E.M.O.L. $=5 \%$.S.M.C. $-p=2,5 \%$

190

Figura 165- VaR - Carteira E.M.O.L. $=5 \% . T . V . E . ~-p=5 \%$

190

Figura $166-$ VaR - Carteira E.M.O.L. $=5 \%$.S.M.C. $-p=5 \%$

Figura 167 - Hill - plot da Carteira A.M.I.U. $(K h=-18,43 \% ; q=6)$

192

Figura 168 - Hill - plot da Carteira A.M.I.U. $(\mathrm{Kh}=-18,86 \% ; q=7)$

192

Figura 169 - Hill - plot da Carteira A.M.I.U. $(\mathrm{Kh}=-18,86 \% ; q=7)$ 192

Figura $170-$ Hill - plot da Carteira A.M.I.U. $(K h=-23,52 \% ; q=2)$ 193

Figura 171 - Hill - plot da Carteira A.M.I.U. $(\mathrm{Kh}=-26,11 \% ; q=3)$ 193

Figura 172 - Hill - plot da Carteira A.M.O.L=0\%

$(\mathrm{Kh}=-14,87 \% ; \mathrm{q}=5)$.

Figura 173 - Hill - plot da Carteira A.M.O.L=0\%

$(\mathrm{Kh}=-14,87 \% ; \mathrm{q}=5)$. 194

Figura 174 - Hill - plot da Carteira A.M.O.L=0\%

$(\mathrm{Kh}=-14,87 \% ; \mathrm{q}=4)$ 194

Figura 175 - Hill - plot da Carteira A.M.O.L=0\%

$(\mathrm{Kh}=-11,42 \% ; \mathrm{q}=2)$

Figura 176 - Hill - plot da Carteira A.M.O.L=0\%

$(\mathrm{Kh}=-11,42 \% ; \mathrm{q}=4)$ 
Figura 177 - Hill - plot da Carteira A.M.O.L=2,5\%

$(\mathrm{Kh}=-20,84 \% ; \mathrm{q}=3)$.

Figura 178 - Hill - plot da Carteira A.M.O.L=2,5\%

$(\mathrm{Kh}=-15,76 \% ; \mathrm{q}=4)$.

Figura 179 - Hill - plot da Carteira A.M.O.L=2,5\% $(\mathrm{Kh}=-15,76 \% ; \mathrm{q}=4)$.

Figura 180- Hill - plot da Carteira A.M.O.L=2,5\%

$$
(K h=-25,42 \% ; q=13) \text {. }
$$

Figura 181- Hill - plot da Carteira A.M.O.L=2,5\%

$(K h=-29,34 \% ; q=14)$.

Figura 182 - Hill - plot da Carteira A.M.O.L=5\%

$(\mathrm{Kh}=-20,84 \% ; \mathrm{q}=3)$

Figura 183 - Hill - plot da Carteira A.M.O.L=5\%

$(\mathrm{Kh}=-17,92 \% ; \mathrm{q}=4)$.

Figura 184 - Hill - plot da Carteira A.M.O.L=5\%

$(\mathrm{Kh}=-17,92 \% ; \mathrm{q}=4)$

Figura 185 - Hill - plot da Carteira A.M.O.L=5\%

$(\mathrm{Kh}=-26,20 \% ; \mathrm{q}=13)$

Figura 186 - Hill - plot da Carteira A.M.O.L=5\%

$(\mathrm{Kh}=-29,79 \% ; \mathrm{q}=10)$

Figura 187 - Hill - plot da Carteira B.M.I.U.

$(\mathrm{Kh}=-34,77 \% ; \mathrm{q}=13)$

Figura 188 - Hill - plot da Carteira A.M.O.L=0\%

$(\mathrm{Kh}=-34,77 \% ; \mathrm{q}=13)$

Figura 189 - Hill - plot da Carteira B.M.I.U.

$(\mathrm{Kh}=-22,87 \% ; \mathrm{q}=4)$. 
Figura 190 - Hill - plot da Carteira B.M.I.U.

$(\mathrm{Kh}=-22,87 \% ; \mathrm{q}=4)$.

Figura 191 - Hill - plot da Carteira B.M.I.U.

$(\mathrm{Kh}=-21,65 \% ; q=3)$

Figura 192 - Hill - plot da Carteira B.M.O.L. $=0 \%$

$(\mathrm{Kh}=-3,87 \% ; \mathrm{q}=2)$

Figura 193 - Hill - plot da Carteira B.M.O.L. $=0 \%$

$(\mathrm{Kh}=--33,20 \% ; \mathrm{q}=23)$.

Figura 194 - Hill - plot da Carteira B.M.O.L.=0\%

$(\mathrm{Kh}=-37,28 \% ; \mathrm{q}=45)$

Figura 195 - Hill - plot da Carteira B.M.O.L. $=0 \%$

$(\mathrm{Kh}=-37,09 \% ; \mathrm{q}=44)$.

201

Figura 196 - Hill - plot da Carteira B.M.O.L. $=0 \%$

$(\mathrm{Kh}=-37,47 \% ; \mathrm{q}=47)$.

201

Figura 197 - Hill - plot da Carteira B.M.O.L.=2,5\%

$(\mathrm{Kh}=-8,44 \% ; \mathrm{q}=3)$.

202

Figura 198 - Hill - plot da Carteira B.M.O.L. $=2,5 \%$

$(\mathrm{Kh}=-9,33 \% ; \mathrm{q}=1)$

Figura 199 - Hill - plot da Carteira B.M.O.L. $=2,5 \%$

$(\mathrm{Kh}=-14,49 \% ; \mathrm{q}=3)$

Figura $200-$ Hill - plot da Carteira B.M.O.L.=2,5\%

$(\mathrm{Kh}=-12,44 \% ; \mathrm{q}=4)$.

203

Figura 201 - Hill - plot da Carteira B.M.O.L.=2,5\%

$(\mathrm{Kh}=-21,72 \% ; \mathrm{q}=17)$ 203

Figura $202-$ Hill - plot da Carteira B.M.O.L. $=5 \%$

$(\mathrm{Kh}=-18,33 \% ; \mathrm{q}=1)$ 
Figura $203-$ Hill - plot da Carteira B.M.O.L. $=5 \%$

$(\mathrm{Kh}=-10,46 \% ; \mathrm{q}=2)$

Figura $204-$ Hill - plot da Carteira B.M.O.L. $=5 \%$

$(\mathrm{Kh}=-10,46 \% ; \mathrm{q}=2)$.

Figura $205-$ Hill - plot da Carteira B.M.O.L. $=5 \%$

$(\mathrm{Kh}=-16,82 \% ; \mathrm{q}=2)$

204

Figura $206-$ Hill - plot da Carteira B.M.O.L.=5\%

$(\mathrm{Kh}=-12,19 \% ; \mathrm{q}=5)$

Figura 207 - Hill - plot da Carteira C.M.I.U.

$(\mathrm{Kh}=-19,73 \% ; \mathrm{q}=5)$

Figura 208 - Hill - plot da Carteira C.M.O.L. $=0 \%$

$(\mathrm{Kh}=-22,38 \% ; \mathrm{q}=5)$

Figura 209 - Hill - plot da Carteira C.M.O.L. $=0 \%$

$(\mathrm{Kh}=-13,91 \% ; \mathrm{q}=3)$

Figura 210 - Hill - plot da Carteira C.M.O.L. $=0 \%$

$(\mathrm{Kh}=-14,59 \% ; \mathrm{q}=3)$

206

Figura 211 - Hill - plot da Carteira C.M.O.L. $=0 \%$

$(\mathrm{Kh}=-12,40 \% ; \mathrm{q}=4)$

Figura 212 - Hill - plot da Carteira C.M.O.L. $=1 \%$

$(\mathrm{Kh}=-18,06 \% ; \mathrm{q}=2)$.

Figura $213-$ Hill - plot da Carteira C.M.O.L. $=1 \%$

$(\mathrm{Kh}=-23,61 \% ; \mathrm{q}=16)$

Figura 214 - Hill - plot da Carteira C.M.O.L. $=1 \%$

$(\mathrm{Kh}=-26,29 \% ; \mathrm{q}=16)$ 
Figura 215 - Hill - plot da Carteira C.M.O.L.=1\%

$(\mathrm{Kh}=-12,78 \% ; \mathrm{q}=1)$

Figura 216 - Hill - plot da Carteira C.M.O.L. $=1 \%$

$(\mathrm{Kh}=-11,43 \% ; \mathrm{q}=1)$

Figura 217 - Hill - plot da Carteira C.M.O.L. $=2,5 \%$

$(\mathrm{Kh}=22,35 \% ; \mathrm{q}=4)$

Figura 218 - Hill - plot da Carteira C.M.O.L.=2,5\%

$(\mathrm{Kh}=14,54 \% ; \mathrm{q}=1)$

Figura 219 - Hill - plot da Carteira C.M.O.L.=2,5\%

$(\mathrm{Kh}=15,75 \% ; \mathrm{q}=7)$

Figura 220 - Hill - plot da Carteira C.M.O.L.=2,5\%

$(\mathrm{Kh}=-12,05 \% ; \mathrm{q}=6)$

209

Figura 221 - Hill - plot da Carteira C.M.O.L. $=2,5 \%$

$(\mathrm{Kh}=-6,82 \% ; \mathrm{q}=3)$

Figura 222 - Hill - plot da Carteira C.M.O.L. $=5 \%$

$(\mathrm{Kh}=-23,65 \% ; \mathrm{q}=, 73)$

210

Figura 223 - . Hill - plot da Carteira C.M.O.L. $=5 \%$

$(\mathrm{Kh}=-20,57 \% ; \mathrm{q}=21)$

Figura $224-$ Hill - plot da Carteira C.M.O.L. $=5 \%$

$(\mathrm{Kh}=-13,50 \% ; \mathrm{q}=2)$.

Figura 225 - Hill - plot da Carteira C.M.O.L. $=5 \%$

$(\mathrm{Kh}=-13,50 \% ; \mathrm{q}=2)$

Figura 226 - Hill - plot da Carteira C.M.O.L. $=5 \%$

$(\mathrm{Kh}=-17,15 \% ; \mathrm{q}=12)$

Figura 227 - Hill - plot da Carteira D.M.I.U.

$(\mathrm{Kh}=-19,73 \% ; \mathrm{q}=5)$ 
Figura 228 - Hill - plot da Carteira D.M.I.U.

$(\mathrm{Kh}=-18,01 \% ; \mathrm{q}=2)$

Figura 229 - Hill - plot da Carteira D.M.I.U.

$(\mathrm{Kh}=-15,76 \% ; q=4)$

Figura 230 - Hill - plot da Carteira D.M.I.U.

$(\mathrm{Kh}=-12,45 \% ; \mathrm{q}=3)$

Figura 231 - Hill - plot da Carteira D.M.I.U.

$(K h=-16,35 \% ; q=10)$

Figura $232-$ Hill - plot da Carteira D.M.O.L. $=0 \%$

$(\mathrm{Kh}=-16,57 \% ; \mathrm{q}=6)$

Figura 233 - Hill - plot da Carteira D.M.O.L. $=0 \%$

$(\mathrm{Kh}=-18,57 \% ; \mathrm{q}=2)$

Figura $234-$ Hill - plot da Carteira D.M.O.L. $=0 \%$

$(\mathrm{Kh}=-15,76 \% ; \mathrm{q}=4)$

Figura 235 - Hill - plot da Carteira D.M.O.L. $=0 \%$

$(\mathrm{Kh}=-12,45 \% ; \mathrm{q}=3)$

214

Figura $236-$ Hill - plot da Carteira D.M.O.L. $=0 \%$

$(\mathrm{Kh}=-16,35 \% ; \mathrm{q}=10)$

Figura 237 - Hill - plot da Carteira D.M.O.L. $=2,5 \%$

$(\mathrm{Kh}=-18,16 \% ; \mathrm{q}=6)$

Figura 238 - Hill - plot da Carteira D.M.O.L.=2,5\%

$(\mathrm{Kh}=-17,28 \% ; \mathrm{q}=9)$

Figura 239 - Hill - plot da Carteira D.M.O.L. $=2,5 \%$

$(\mathrm{Kh}=-15,50 \% ; \mathrm{q}=8)$

Figura 240 - Hill - plot da Carteira D.M.O.L.=2,5\%

$(\mathrm{Kh}=-16,18 \% ; \mathrm{q}=11)$ 
Figura 241 - Hill - plot da Carteira D.M.O.L. $=2,5 \%$

$(\mathrm{Kh}=-13,59 \% ; \mathrm{q}=2)$

Figura $242-$ Hill - plot da Carteira D.M.O.L. $=5 \%$

$(\mathrm{Kh}=-18,75 \% ; \mathrm{q}=8)$

Figura $243-$ Hill - plot da Carteira D.M.O.L. $=5 \%$

$(\mathrm{Kh}=-17,59 \% ; \mathrm{q}=9)$

Figura 244 - Hill - plot da Carteira D.M.O.L. $=5 \%$

$(\mathrm{Kh}=-16,03 \% ; \mathrm{q}=8)$

Figura 245 - Hill - plot da Carteira D.M.O.L. $=5 \%$

$(\mathrm{Kh}=-16,93 \% ; \mathrm{q}=10)$

Figura 246 - Hill - plot da Carteira D.M.O.L. $=5 \%$

$(\mathrm{Kh}=-13,23 \% ; \mathrm{q}=2)$

218

Figura 247 - Hill - plot da Carteira E.M.I.U.

$(\mathrm{Kh}=-9,03 \% ; \mathrm{q}=3)$

Figura 248 - Hill - plot da Carteira E.M.I.U.

$(\mathrm{Kh}=-19,72 \% ; \mathrm{q}=5)$.

219

Figura 249 - Hill - plot da Carteira E.M.I.U.

$(\mathrm{Kh}=-13,66 \% ; \mathrm{q}=7)$

Figura 250 - Hill - plot da Carteira E.M.I.U.

$(\mathrm{Kh}=-14,81 \% ; \mathrm{q}=11)$

Figura 251 - Hill - plot da Carteira E.M.I.U.

$(\mathrm{Kh}=-15,27 \% ; q=1)$

220

Figura $252-$ Hill - plot da Carteira E.M.O.L. $=0 \%$

$(\mathrm{Kh}=-9,03 \% ; \mathrm{q}=3)$

Figura 253 - Hill - plot da Carteira E.M.O.L. = 0\%

$(\mathrm{Kh}=-18,73 \% ; \mathrm{q}=4)$ 
Figura $254-$ Hill - plot da Carteira E.M.O.L. $=0 \%$

$(\mathrm{Kh}=-15,0 \% ; \mathrm{q}=9)$

Figura $255-$ Hill - plot da Carteira E.M.O.L. $=0 \%$

$(\mathrm{Kh}=-17,42 \% ; q=10)$

Figura $256-$ Hill - plot da Carteira E.M.O.L. $=0 \%$

$(\mathrm{Kh}=-16,87 \% ; \mathrm{q}=12)$

Figura 257 - Hill - plot da Carteira E.M.O.L. $=2,5 \% \%$

$(\mathrm{Kh}=-2,12 ; \mathrm{q}=2)$.

222

Figura 258 - Hill - plot da Carteira E.M.O.L. $=2,5 \%$

$(\mathrm{Kh}=-10,87 \% ; \mathrm{q}=3)$

222

Figura 259 - Hill - plot da Carteira E.M.O.L. $=2,5 \%$

$(\mathrm{Kh}=-15,65 \% ; \mathrm{q}=2)$

222

Figura 260 - Hill - plot da Carteira E.M.O.L. $=2,5 \%$

$(\mathrm{Kh}=-14,24 \% ; \mathrm{q}=3)$

Figura $261-$ Hill - plot da Carteira E.M.O.L. $=2,5 \%$

$(\mathrm{Kh}=-16,94 \% ; \mathrm{q}=17)$

223

Figura $262-$ Hill - plot da Carteira E.M.O.L. $=5 \%$

$(\mathrm{Kh}=-13,20 \% ; \mathrm{q}=2)$

Figura $263-$ Hill - plot da Carteira E.M.O.L. $=5 \%$

$(\mathrm{Kh}=-14,29 \% ; \mathrm{q}=2)$

Figura $264-$. Hill - plot da Carteira E.M.O.L. $=5 \%$

$(\mathrm{Kh}=-15,71 \% ; \mathrm{q}=2)$

224

Figura $265-$ Hill - plot da Carteira E.M.O.L. $=5 \%$

$(\mathrm{Kh}=-14,13 \% ; \mathrm{q}=3)$

224

Figura $266-$ Hill - plot da Carteira E.M.O.L. $=5 \%$

$(\mathrm{Kh}=-17,14 \% ; \mathrm{q}=16))$ 


\section{Lista de tabelas}

Tabela 1 - Tipos de erro do modelo 47

Tabela 2 - Distribuição de frequências de cotação de uma moeda $\quad 77$

Tabela 3 - Geração de números aleatórios 79

Tabela 4 - 15 ações mais negociadas (1994.1 - 1996.2) 85

Tabela 5 - Otimização no contexto M.I.U. para as

15 ações mais negociadas (1994.2 - 1996.2) 85

Tabela 6 - Otimização no contexto M.O.L. = $0 \%$ para as

15 ações mais negociadas (1994.2 - 1996.2) 87

Tabela 7 - - Otimização no contexto M.O.L. $=2,5 \%$ para as

15 ações mais negociadas (1994.2 - 1996.2)

Tabela 8 - - Otimização no contexto M.O.L. = 5\% para as

15 ações mais negociadas (1994.2 - 1996.2)

Tabela 9 - Otimização no contexto do M.I.U. e M.O. para as 15 ações mais negociadas no período 1994.2 - 1996.2.

Tabela 10 - 15 ações mais negociadas (1997.1 - 1998.2)

Tabela 11 - Otimização no contexto M.I.U. para as

15 ações mais negociadas (1997.1 - 1998.2)

Tabela 12 - Otimização no contexto M.O.L. = 0\% para as

15 ações mais negociadas (1997.1 - 1998.2)

Tabela 13 - Otimização no contexto M.O.L. $=2,5 \%$ para as 15 ações mais negociadas (1997.1 - 1998.2) 
Tabela 14 - Otimização no contexto M.O.L. = 5\% para as

15 ações mais negociadas (1997.1 - 1998.2)

Tabela 15 - Otimização no contexto do M.I.U. e M.O. para as 15 ações mais negociadas no período 1997.1 - 1998.2.

Tabela 16 - 15 ações mais negociadas (1999.1 - 2000.2)

Tabela 17 - Otimização no contexto M.I.U. para as

15 ações mais negociadas (1999.1 - 2000.2)

Tabela 18 - Otimização no contexto M.O.L. $=0 \%$ para as 15 ações mais negociadas (1999.1 - 2000.2)

Tabela 19 - Otimização no contexto M.O.L. = 2,5\% para as 15 ações mais negociadas (1999.1 - 2000.2)

Tabela 20 - Otimização no contexto M.O.L. $=5 \%$ para as 15 ações mais negociadas (1999.1 - 2000.2)

Tabela 21 - Otimização no contexto M.I.U. e M.O. para as 15 ações mais negociadas (1999.1 - 2000.2)

Tabela 22 - 15 ações mais negociadas (1999.1 - 2000.2)

Tabela 23 - Otimização no contexto M.I.U. para as 15 ações mais negociadas (2001.1 - 2002.2)

Tabela 24 - Otimização no contexto M.O.L. $=0 \%$ para as 15 ações mais negociadas (2001.1 - 2002.2)

Tabela 25 - Otimização no contexto M.O.L. = 2,5\% para as 15 ações mais negociadas (2001.1 - 2002.2)

Tabela 26 - Otimização no contexto M.O.L. = 5\% para as 15 ações mais negociadas (2001.1 - 2002.2) 
Tabela 27 - Otimização no contexto do M.I.U. e M.O. para as 15 ações mais negociadas no período 2001.1 - 2002.2

Tabela 28 - 15 ações mais negociadas (2003.1 - 2004.2)

Tabela 29 - Otimização no contexto M.I.U. para as

15 ações mais negociadas (2003.1 - 2004.2)

Tabela 30 - Otimização no contexto M.O.L. = 0\% para as

15 ações mais negociadas (2003.1 - 2004.2)

Tabela 31 - Otimização no contexto M.O.L. $=2,5 \%$ para as 15 ações mais negociadas (2003.1 - 2004.2)

Tabela 32 - Otimização no contexto M.O.L. = 5\% para as 15 ações mais negociadas (2003.1 - 2004.2)

Tabela 33 - Otimização no contexto do M.I.U. e M.O. para as 15 ações mais negociadas no período 2003.1 - 2004.2

Tabela 34 - Otimização no contexto do M.I.U. e M.O. para as 15 ações mais negociadas no período 1994.2 - 1996.2

Tabela 35 -Análise do desempenho das simulações

T.V.E. e S.M.C. para a Carteira A.M.I.U

Tabela 36 -Análise do desempenho das simulações

T.V.E. e S.M.C. para a Carteira A.M.O.L. $=0 \%$.

Tabela 37 -Análise do desempenho das simulações

T.V.E. e S.M.C. para a Carteira A.M.O.L. $=2,5 \%$

Tabela 38 -Análise do desempenho das simulaçõe

T.V.E. e S.M.C. para a Carteira A.M.O.L. $=5 \%$

Tabela 39 -Análise do desempenho das simulações 
Tabela 40 -Análise do desempenho das simulações

T.V.E. e S.M.C. para a Carteira B.M.O.L. $=0 \%$

Tabela 41 -Análise do desempenho das simulações

T.V.E. e S.M.C. para a Carteira B.M.O.L. $=2,5 \%$

Tabela 42 -Análise do desempenho das simulações

T.V.E. e S.M.C. para a Carteira B.M.O.L. $=5 \%$

Tabela 43 -Análise do desempenho das simulações

T.V.E. e S.M.C. para a Carteira C.M.I.U

Tabela 44 -Análise do desempenho das simulações

T.V.E. e S.M.C. para a Carteira C.M.O.L. $=0 \%$

Tabela 45 -Análise do desempenho das simulações

T.V.E. e S.M.C. para a Carteira C.M.O.L. $=2,5 \%$

Tabela 46 -Análise do desempenho das simulações

T.V.E. e S.M.C. para a Carteira C.M.O.L. $=5 \%$

Tabela 47 -Análise do desempenho das simulações T.V.E. e S.M.C. para a Carteira D.M.I.U.

Tabela 48 -Análise do desempenho das simulações

T.V.E. e S.M.C. para a Carteira D.M.O.L. $=0 \%$.

Tabela 49 -Análise do desempenho das simulações

T.V.E. e S.M.C. para a Carteira D.M.O.L. $=2,5 \%$

Tabela 50 -Análise do desempenho das simulações

T.V.E. e S.M.C. para a Carteira D.M.O.L. $=5 \%$

Tabela 51 -Análise do desempenho das simulações

T.V.E. e S.M.C. para a Carteira E.M.I.U

Tabela 52 -Análise do desempenho das simulações

T.V.E. e S.M.C. para a Carteira E.M.O.L. $=0 \%$. 
Tabela 53 -Análise do desempenho das simulações

T.V.E. e S.M.C. para a Carteira E.M.O.L. $=2,5 \%$.

Tabela 54 -Análise do desempenho das simulações

T.V.E. e S.M.C. para a Carteira E.M.O.L. $=5 \%$.

Tabela 55 - Otimização no contexto do M.I.U. e M.O.

para as 15 ações mais negociadas no período 1994.2 - 1996.2

Tabela 56 - Otimização no contexto do M.I.U. e M.O.

para as 15 ações mais negociadas no período 1997.1 - 1998.2

Tabela 57 - Otimização no contexto do M.I.U. e M.O.

para as 15 ações mais negociadas no período 1999.1 - 2000.2

Tabela 58 - Otimização no contexto do M.I.U. e M.O.

para as 15 ações mais negociadas no período 2001.1 - 2002.2

Tabela 59 - Otimização no contexto do M.I.U. e M.O.

para as 15 ações mais negociadas no período 2003.1 - 2004.2

Tabela 60 - Análise do desempenho das Simulações

T.V.E. e S.M.C. para a Carteira A constituída por

Modelo de Índice Único

Tabela 61 - Análise do desempenho das Simulações

T.V.E. e S.M.C. para a Carteira A constituída por Medida Ômega, com $L=0 \%$

Tabela 62 - Análise do desempenho das Simulações T.V.E. e S.M.C. para a Carteira A constituída por Medida Ômega, $\operatorname{com} \mathrm{L}=2,5 \%$

Tabela 63 - Análise do desempenho das Simulações T.V.E. e S.M.C. para a Carteira A constituída por Medida Ômega, com $L=5 \%$ 
Tabela 64 - Análise do desempenho das Simulações

T.V.E. e S.M.C. para a Carteira B constituída por Modelo de Índice Único

Tabela 65 - Análise do desempenho das Simulações

T.V.E. e S.M.C. para a Carteira B constituída por Medida Ômega, com $L=0 \%$

Tabela 66 - Análise do desempenho das Simulações

T.V.E. e S.M.C. para a Carteira B constituída por Medida Ômega, com $L=2,5 \%$

Tabela 67 - Análise do desempenho das Simulações

T.V.E. e S.M.C. para a Carteira B constituída por Medida Ômega, com $L=5 \%$

Tabela 68 - Análise do desempenho das Simulações

T.V.E. e S.M.C. para a Carteira C constituída por Modelo de Índice Único

Tabela 69 - Análise do desempenho das Simulações

T.V.E. e S.M.C. para a Carteira C constituída por Medida Ômega, com $L=0 \%$

Tabela 70 - Análise do desempenho das Simulações

T.V.E. e S.M.C. para a Carteira C constituída por Medida Ômega, $\operatorname{com} \mathrm{L}=2,5 \%$

Tabela 71 - Análise do desempenho das Simulações

T.V.E. e S.M.C. para a Carteira C constituída por Medida Ômega, com $L=5 \%$

Tabela 72 - Análise do desempenho das Simulações

T.V.E. e S.M.C. para a Carteira D constituída por Modelo de Índice Único 
Tabela 73 - Análise do desempenho das Simulações

T.V.E. e S.M.C. para a Carteira D constituída por Medida Ômega, com $L=0 \%$

Tabela 74 - Análise do desempenho das Simulações

T.V.E. e S.M.C. para a Carteira D constituída por Medida Ômega, com $\mathrm{L}=2,5 \%$

Tabela 75 - Análise do desempenho das Simulações

T.V.E. e S.M.C. para a Carteira D constituída por Medida Ômega, com $L=5 \%$

Tabela 76 - Análise do desempenho das Simulações

T.V.E. e S.M.C. para a Carteira E constituída por Modelo de Índice Único

Tabela 77 - Análise do desempenho das Simulações

T.V.E. e S.M.C. para a Carteira E constituída por Medida Ômega, com $L=0 \%$

Tabela 78 - Análise do desempenho das Simulações

T.V.E. e S.M.C. para a Carteira E constituída por Medida Ômega, com $L=2,5 \%$

Tabela 79 - Análise do desempenho das Simulações

T.V.E. e S.M.C. para a Carteira E constituída por Medida Ômega, com $L=5 \%$

Tabela 80 - Análise do desempenho das Simulações

T.V.E. e S.M.C. para níveis de confiança do VaR

Tabela 81 - : Análise do desempenho das Simulações

T.V.E. e S.M.C. para níveis de confiança do VaR

(excluindo-se a Carteira E) 